Supporting Information

\title{
Folded Chain Lamellae of Dynamic Helical Poly(phenylacetylene) in Hexagonal Columnar Phase
}

\author{
Yan-Fang Zhang, ${ }^{\dagger}$ Xu Chen, ${ }^{\dagger}$ Xiao-Song Yu,,$^{\dagger}$ Jia-Xin Chen, ${ }^{\dagger}$ Ming-Qiu Hu,,$^{\dagger}$ Bo-Yuan Zheng,,$^{\dagger}$ \\ Yi-Xin Liu, ** Shuang Yang, ${ }^{*}+$ and Er-Qiang Chen $*+$
}

${ }^{\dagger}$ Beijing National Laboratory for Molecular Sciences, Key Laboratory of Polymer Chemistry and Physics of Ministry of Education, Center for Soft Matter Science and Engineering, College of Chemistry, Peking University, Beijing, 100871, China.

\$State Key Laboratory of Molecular Engineering of Polymers, Department of Macromolecular Science, Fudan University, Shanghai, 200438, China.

Corresponding Author

*Email: eqchen@pku.edu.cn (E. Q. C.)

*Email: shuangyang@pku.edu.cn (S. Y.)

*Email: lyx@fudan.edu.cn (Y. X. L) 


\section{Table of Content}

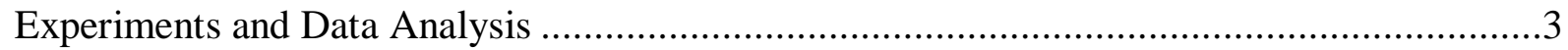

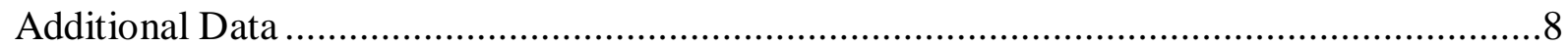

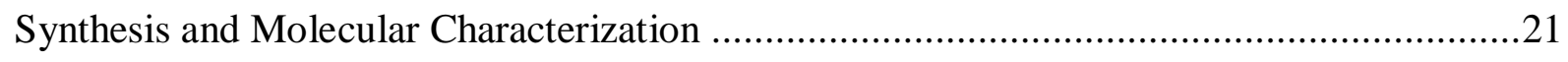

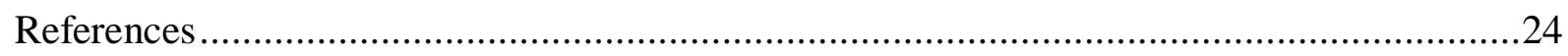




\section{Experiments and Data Analysis}

\section{Reagents and materials}

Unless otherwise noted, all reagents and solvents used in the experiments were obtained from commercial sources without further purification. Tetrahydrofuran (THF) and diisopropylamine (DIPA) were distilled and dried over calcium hydride and potassium hydroxide, respectively. The catalyst used for polymerization of the phenylacetylene monomer, $\mathrm{Rh}(\mathrm{nbd})\left[\mathrm{B}\left(\mathrm{C}_{6} \mathrm{H}_{5}\right)_{4}\right]$, was prepared according to a previously reported method. ${ }^{1}$

\section{Instruments and measurements}

Nuclear magnetic resonance $\left({ }^{1} \mathrm{H} \mathrm{NMR}, 400 \mathrm{MHz}\right)$ spectra were obtained on a Bruker ARX400 spectrometer. Chemical shift was relative to tetramethylsilane (TMS) as the internal standard.

Mass spectra were obtained on a Bruker Solarix XR mass spectrometer.

Elemental analyses were performed on a Vario EL elementar.

Gel permeation chromatography (GPC) was carried out on a Waters 515 GPC instrument using THF as an eluent. The GPC calibration curve was obtained with linear polystyrene standards.

Circular dichroism (CD) spectra were recorded on a benchtop JASCO J-810 spectrometer with a wide beam (beam area in the $\mathrm{cm}^{2}$ range). The solution samples for CD measurement were prepared by dissolving $\mathbf{P 1}$ in chloroform. The film samples for CD measurement were prepared by drop-casting on the quartz substrate from the chloroform solution $(5 \mathrm{mg} / \mathrm{mL})$ of $\mathbf{P 1}$, subsequently by volatilization of chloroform solvent at room temperature and then drying in a vacuum oven.

Both static light scattering (SLS) and dynamic light scattering (DLS) were performed using a commercialized spectrometer from Brookhaven Instrument Corporation (BI-200SM Goniometer, Holtsville, NY). A vertically polarized, $17 \mathrm{~mW}$ He-Ne laser (Research ElectroOptics, Inc., Boulder, CO) operating at $633 \mathrm{~nm}$ was used as the light source. The concentrations of a series of cyclohexane solutions used in SLS measurements were 0.050, 0.096, 0.21, 0.35 and $0.52 \mathrm{mg} / \mathrm{mL}$, respectively. The specific refractive index increment $(\mathrm{d} n / \mathrm{d} c)$ of P1 cyclohexane solutions was measured using BI-DNDC differential refractometer.

One-dimensional (1D), two-dimensional (2D) and grazing-incidence (GI) X-ray diffraction (XRD) of the samples were recorded by employing a Ganesha system (SAXSLAB, 
U.S) equipped with a multilayer focused $\mathrm{Cu} \mathrm{K \alpha}$ radiation as the $\mathrm{X}$-ray source (Genix3D $\mathrm{Cu}$ ULD) and a semiconductor detector (Pilatus 300 K, DECTRIS, Swiss). The scattering peak positions were calibrated with $\mathrm{LaB}_{6}$ for the wide-angle region and silver behenate for the smallangle region, respectively. A Linkam TST350 stage was utilized to study the structure evolution as a function of temperature. The heating and cooling rates in the experiments were $10{ }^{\circ} \mathrm{C} / \mathrm{min}$. To obtain samples for GI-XRD experiment, a drop of $5 \mathrm{mg} / \mathrm{mL}$ cyclohexane solution of P1 was spin-cast onto the substrate of a silicon wafer.

Dimension Icon atomic force microscopy (AFM, Bruker Nano) was used at room temperature. PeakForce tapping mode was applied in the experiments using SCANASYSTAIR probes (tip radius: $\sim 2 \mathrm{~nm}$; spring 205 constant: $\sim 0.4 \mathrm{~N} / \mathrm{m}$; frequency: $\sim 70 \mathrm{kHz}$ ). To obtain samples for AFM experiment, a drop of $5 \mathrm{mg} / \mathrm{mL}$ cyclohexane solution of P1 was spin-cast onto the substrate of a silicon wafer. The rotate speed was $3000 \mathrm{rad} / \mathrm{s}$. All the prepared samples were dried in a vacuum oven at room temperature for $24 \mathrm{~h}$ before annealing. The film thickness of $\mathbf{P 1}$ was about $50 \mathrm{~nm}$ measured by AFM.

\section{Solution Light Scattering}

Static solution light scattering (SLS). Angular dependence of the excess absolute timeaveraged scattered intensity of dilute polymer solutions, known as the Rayleigh ratio $R_{\mathrm{vv}}(\theta)$, was measured by SLS. The weight-averaged molar mass $\left(M_{\mathrm{w}}\right)$ and the root-mean-square radius of gyration $\left(R_{\mathrm{g}}\right)$ can be obtained according to the following equations,

$$
\frac{K c}{R_{V V}(\theta)}=\frac{1}{M_{w}}\left(1+\frac{1}{3} q^{2} R_{g}^{2}\right)+2 A_{2} c
$$

with $K=\frac{4 \pi^{2} n^{2}(d n / d c)^{2}}{N_{A} \lambda^{4}}$ and $q=\frac{4 \pi n \sin (\theta / 2)}{\lambda}$, wherein $N_{\mathrm{A}}, n, \mathrm{~d} n / \mathrm{d} c$, and $\lambda$ are the Avogadro's number, solvent refractive index, specific refractive index increment, and the wavelength of light in a vacuum, respectively.

The persistence length $l_{\mathrm{P}}$ can be obtained by solving the following equation,

$$
R_{g}{ }^{2}=\frac{1}{3} l_{P} l_{C}-l_{P}{ }^{2}+2 \frac{l_{P}{ }^{3}}{l_{C}}\left(1-\frac{l_{P}}{l_{C}}\left[1-\exp \left(-\frac{l_{P}}{l_{C}}\right)\right]\right)
$$

where $l_{\mathrm{C}}$ is the contour length of the polymer chain.

Dynamic solution light scattering (DLS). The intensity-intensity time correlation function $G^{(2)}(\tau)$ in the self-beating mode was measured by DLS. A Laplace inversion program, CONTIN, was used to process the data to obtain the line width distribution and the diffusion coefficient. The diffusion coefficient $D$ can be further converted into the hydrodynamic radius $R_{\mathrm{h}}$ by using the Stokes-Einstein equation, 


$$
D=\frac{k_{B} T}{6 \pi \eta R_{h}}
$$

where $k_{\mathrm{B}}, T$, and $\eta$ are the Boltzmann constant, the absolute temperature, and the viscosity of the solvent, respectively.

\section{Reconstruction of Relative Electron Density Distribution}

Based on XRD data, the reconstruction of relative electron density distribution in real space is carried out using the following formula for three-dimensional (3D) Fourier transformation,

$$
\rho(x, y, z)-\rho_{0}=\sum_{h k l} F(h k l) \exp [i 2 \pi(h x+k y+l z)]
$$

where $\rho$ is the average electron density, $x, y$ and $z$ are the fractional coordinates of a point in the unit cell. $F(h k l)$ is the complex structure factor and its modulus is related to the diffraction intensity $I(h k l)$ by $|F(h k l)|=\sqrt{I(h k l)}$. Note that diffraction intensities need to be multiplicity corrected. In addition, the summation is executed over all possible integer combinations of $(h k l)$ except for (000).

If the lattice is chosen as centrosymmetric, the structure factor becomes real and is given by $F(h k l)= \pm|F(h k l)|$, with corresponding possible phase of $0(+)$ or $\pi(-)$. Then the electron density can be expressed as,

$$
\rho(x, y, z)-\rho_{0}=\sum_{h k l} \pm \sqrt{I(h k l)} \cos [i 2 \pi(h x+k y+l z)]
$$

If we consider a two-dimensional (2D) electron density distribution, then the third component $z$ is absent and all calculations are performed in $(x, y)$ space. For a columnar liquid crystalline system, we assume that the column axis is along the $z$-axis.

The electron density distribution was calculated using the suitable phase combinations for the corresponding reflections. In order to select the proper phases for the diffractions, we used a trial-and-error approach. All the possible phase combinations were used to reconstruct the electron density distributions. The "correct" distribution was then selected based on the quality of the electron density map obtained, aided by prior physical and chemical knowledge of the system.

For P1, the 2D electron density map was calculated based on the four diffractions with the $q$-ratio of $1: \sqrt{3}: \sqrt{4}: \sqrt{7}$ in the low angle region of the XRD profile, which are (10), (11), (20) and (21) diffraction of the hexagonal lattice, respectively. The phases of the four diffractions were selected to be,,+++ and - , respectively. 


\section{Molecular Mechanics Simulation}

Molecular mechanics simulations were performed using Materials Studio software package 8.0 (Accelrys Software Inc.). Geometry optimization, energy minimization, anneal and dynamics calculation were carried out through the Forcite module with the Condensed-phase Optimized Molecular Potentials for Atomistic Simulation Studies (COMPASS) force field. The convergence level for optimization was set to be ultrafine.

To simulate the $\mathbf{P 1}$ with helical conformation, single chains of $\mathbf{P 1}$ with 36 repeating units were built through a head-to-tail arrangement with various connecting dihedral angles. The initial dihedral angles were chosen in the range of $30-180^{\circ}$. After geometry optimization, dynamics and energy minimization, the total energy, chain lengths and the diameters of the oligomer chains were obtained.

To simulate the $\mathbf{P 1}$ with fold conformations, single chains of $\mathbf{P 1}$ with 100 repeating units were built through a head-to-tail arrangement with the initial dihedral angle of $180^{\circ}$. After several cycles of geometry optimization, anneal and dynamics calculation, the dihedral angles of different repeating units varied, leading to some fold conformations of P1 single chains. Afterwards, energy optimization for the folded $\mathbf{P 1}$ strands was carried out at $298 \mathrm{~K}$.

\section{Data Analysis of Ostwald Ripening}

We carried out quasi-in-situ AFM experiments to monitor the lamellar growth of P1. We consider that after solvent elimination, P1 chains in the spin cast film collapse, and the helical segments could condense together to form clusters pervasively. However, due to the limited resolution of AFM, the clusters could not be detected. Furthermore, during the lamellar growth, the lamellar nuclei were also hardly observed under AFM. In this case, to analyze the growth kinetics of the P1 lamellae, only parts of lamellae were selected for our statistics. The principle of the selection is shown in the following. Firstly, the lamella should be intact in our AFM view during the whole monitoring period. Then the growth of the lamella should be free from merging with other lamellae. Finally, the prolongation, i.e., the lateral growth of the lamella should be free towards both sides. While the growth was easily hindered at the later stage of amorphous-to-liquid crystal transition of $\mathbf{P 1}$ in the thin film, this free growth were well observed at least for the first four AFM images.

Excluding all lamellae that did not meet above requirements, we finally picked several lamellae and investigate the kinetics of their growth. These selected lamellae had relatively large sizes and thus would not vanish in the Ostwald ripening process. The average size of the 
selected lamellae is obviously larger than the average size of all the particles including the less stable clusters and lamellar nuclei. Here, we present a proof that the growth of these selected lamellae still follows the same Ostwald ripening kinetics, as long as the entire family of particles follow the Ostwald ripening kinetics, i.e. $R \sim t^{1 / 3}$ ( $R$ denotes the length of the lamella, i.e., $W$ in the main text).

In an Ostwald ripening, the size distribution function is given by ${ }^{2}$

$$
f(R, t)=\bar{R}^{-4}(t) g(z)
$$

where $\bar{R}$ denotes the average size of all particles in the system, and $g(z)$ is a function of $z=$ $R / \bar{R}$ which is independent of $t$. With the average size of lamellae whose sizes are larger than $\bar{R}$ denoted as $\bar{R}_{+}$, by definition it takes the form

$$
\bar{R}_{+}=\frac{\int_{\bar{R}}^{\infty} R f(R) \mathrm{d} R}{\int_{\bar{R}}^{\infty} f(R) \mathrm{d} R}
$$

The numerator can be rewritten as

$$
\int_{\bar{R}}^{\infty} R f(R) \mathrm{d} R=\int_{\bar{R}}^{\infty} R \bar{R}^{-4}(t) g(z) \mathrm{d} R=\bar{R}^{-2} \int_{\bar{R}}^{\infty} \frac{R}{\bar{R}} g(z) \mathrm{d} \frac{R}{\bar{R}}=\bar{R}^{-2} \int_{1}^{\infty} z g(z) \mathrm{d} z
$$

Similarly, the denominator is reduced to be

$$
\int_{\bar{R}}^{\infty} f(R) \mathrm{d} R=\int_{\bar{R}}^{\infty} \bar{R}^{-4}(t) g(z) \mathrm{d} R=\bar{R}^{-3} \int_{1}^{\infty} g(z) \mathrm{d} z
$$

Then we have

$$
\bar{R}_{+}=\bar{R} \frac{\int_{1}^{\infty} z g(z) \mathrm{d} z}{\int_{1}^{\infty} g(z) \mathrm{d} z}
$$

As $z$ is independent of $t$, we arrives at the final conclusion:

$$
\bar{R}_{+} \sim \bar{R} \sim t^{1 / 3}
$$




\section{Additional Data}

a)

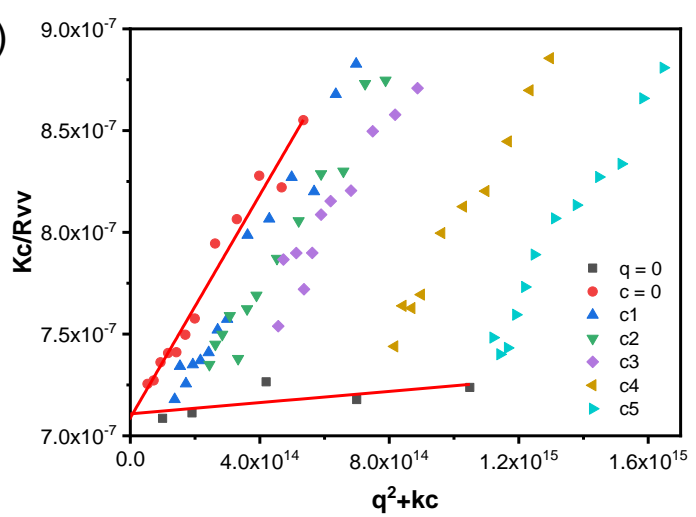

\begin{tabular}{cccccc}
\hline Sample & $\begin{array}{c}\mathrm{d} n / \mathrm{d} c \\
(\mathrm{~mL} / \mathrm{g})\end{array}$ & $\begin{array}{c}\mathrm{M}_{w} \\
\left(10^{4} \mathrm{~g} \cdot \mathrm{mol}^{-1}\right)\end{array}$ & $\begin{array}{c}\mathrm{R}_{g} \\
(\mathrm{~nm})\end{array}$ & $\begin{array}{c}\mathrm{R}_{h} \\
(\mathrm{~nm})\end{array}$ & $\begin{array}{c}l_{p} \\
(\mathrm{~nm})\end{array}$ \\
\hline $\mathrm{P} 1$ & 0.1425 & 141 & 34 & 15 & 6 \\
\hline
\end{tabular}

b)

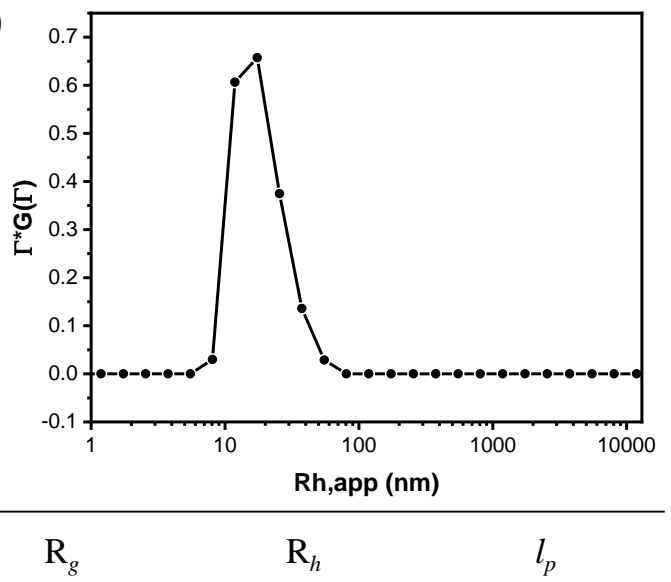

Figure S1. Solution light scattering results of P1. a) Zimm-plot of P1 solution with different concentrations ( $\mathrm{c} 1=0.050, \mathrm{c} 2=0.096, \mathrm{c} 3=0.21, \mathrm{c} 4=0.35, \mathrm{c} 5=0.52 \mathrm{mg} / \mathrm{mL})$. b) Distribution of the hydrodynamic radius $\left(R_{\mathrm{h}}\right)$ of $\mathbf{P 1}$ measured by DLS. The table lists specific refractive index increment $(\mathrm{d} n / \mathrm{d} c)$ of $\mathbf{P 1}$ solution, calculated weight-averaged molar mass $\left(M_{\mathrm{w}}\right)$, rootmean-square radius of gyration $\left(R_{\mathrm{g}}\right)$, hydrodynamic radius $\left(R_{\mathrm{h}}\right)$, and persistence length $\left(l_{\mathrm{P}}\right)$ of $\mathbf{P 1}$ in dilute solutions. The ratio of $R_{\mathrm{g}} / R_{\mathrm{h}}$ is $\sim 2.3$, indicating that as a whole the $\mathbf{P 1}$ chain is rodlike rather than a random coil in the solution. Solvent: cyclohexane. 


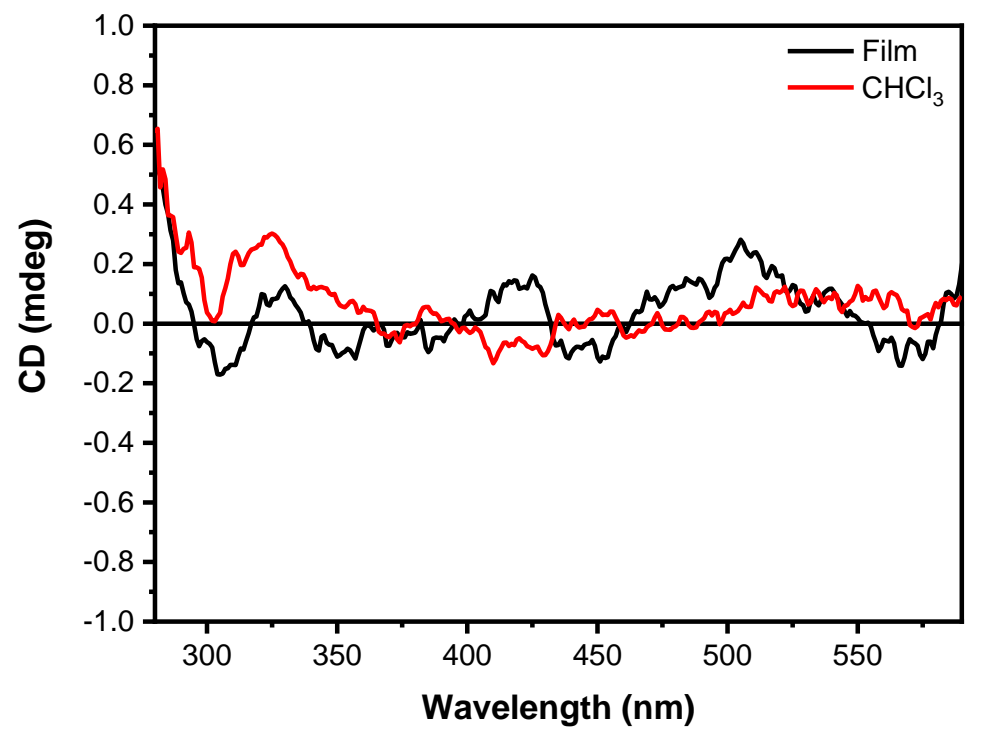

Figure S2. CD spectra of $\mathbf{P 1}$ in chloroform and at the film state. For both the solution and the film, the CD signals fluctuated around zero, showing that there was no excess of a singlehanded helix with either right- or left-handedness. 
a)

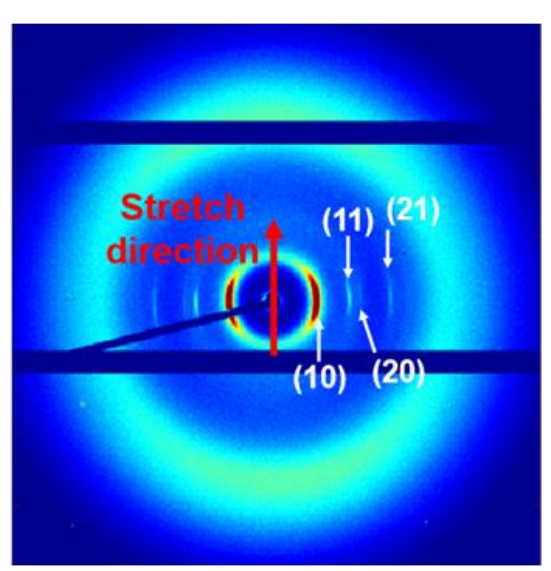

b)

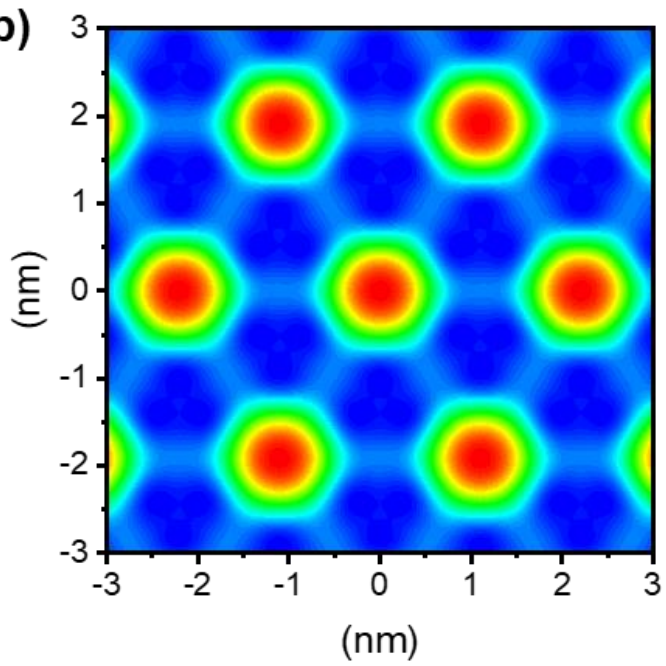

c)

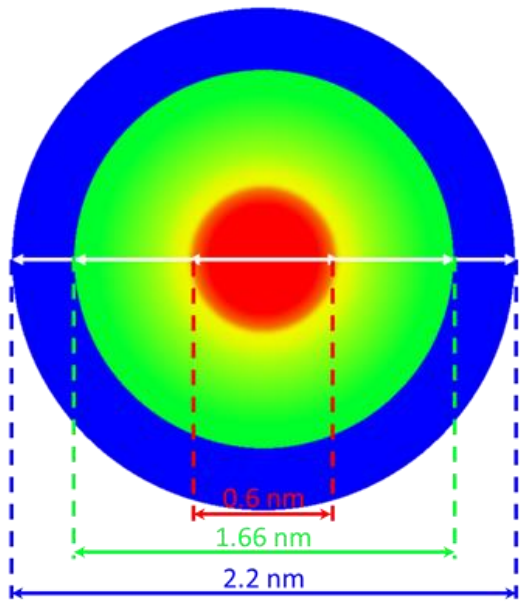

Figure S3. a) 2D XRD pattern of the oriented P1. The oriented sample was prepared by mechanical stretching of a P1 specimen at RT followed by annealing at $120{ }^{\circ} \mathrm{C}$. b) Reconstructed electron density map of $\mathbf{P 1}$ in $\mathrm{Col}_{\mathrm{H}}$ phase. The electron density map was calculated based on the four low angle diffractions with the $q$-ratio of $1: \sqrt{3}: \sqrt{4}: \sqrt{7}$ in the 1D XRD profile, which are assigned as (10), (11), (20) and (21) diffraction, respectively. The phases of the four diffractions are selected to be,,+++ , and - , respectively. c) Schematic top view of $\mathbf{P 1}$ supramolecular column with a core-shell-corona structure. The red, green, and blue regions represent the polyacetylene backbone, benzene rings and ester bonds, and alkyl tails, respectively. 
a)

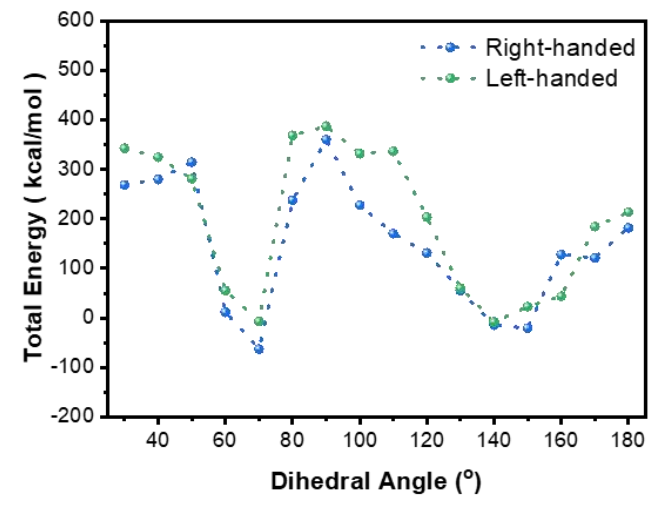

b) Side View

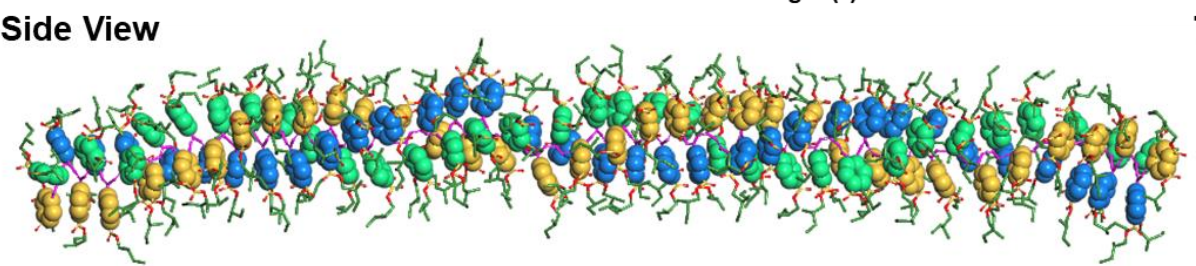

Top View

c)

\section{Side View}

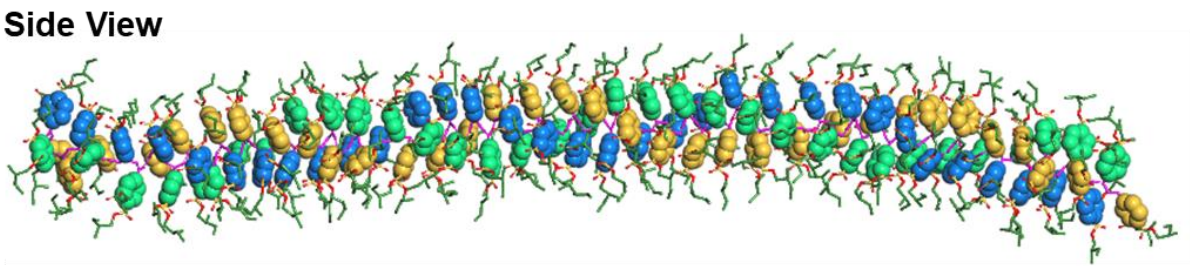

Top View

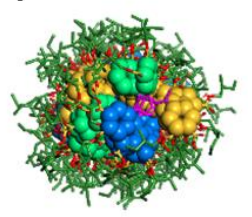

d) Side View

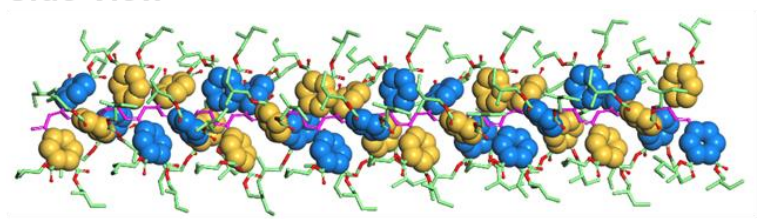

Top View

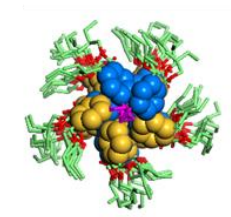

Figure S4. a) Total energy of $\mathbf{P 1}$ single chain of 36 repeating units with different dihedral angles. b) Side and top views of the left-handed helix P1 with 100 repeating units with dihedral angle of $70^{\circ}$. c) Side and top views of the right-handed helix P1 with 100 repeating units with dihedral angle of $70^{\circ}$. d) Side and top views of the right-handed helix P1 with 36 repeating units with dihedral angle of $140^{\circ}$.

It can be seen that the energies of right- and left-handed helices of $\mathbf{P 1}$ have almost the same dihedral angle dependence. The $\mathbf{P 1}$ chain with the cis-cisoidal conformation possesses the lowest enery at the dihedral angle of $70^{\circ}$. Another energy minimum situates at dihedral angle around $140^{\circ}$, corresponding to a cis-transoidal conformation. In comparison with that of the cis-cisoidal conformation with the dihedral angle of $70^{\circ}$, the $c i s$-transoidal molecular cylinder with the dihedral angle of $140^{\circ}$ has the periphery not evenly covered by the liquid-like alkyl tails. In this case, the surface-undulated molecular cylinder cannot be packed closely in the hexagonal columnar phase. 
a)

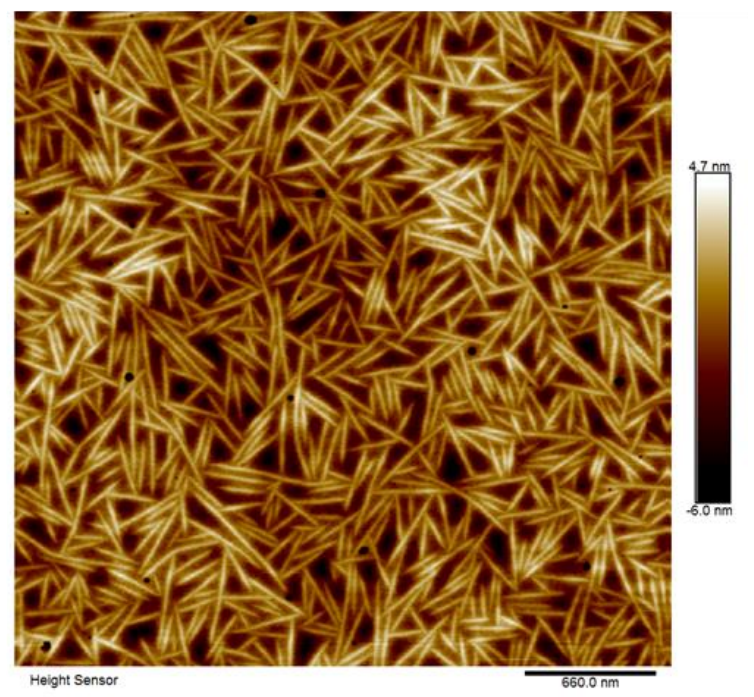

b)

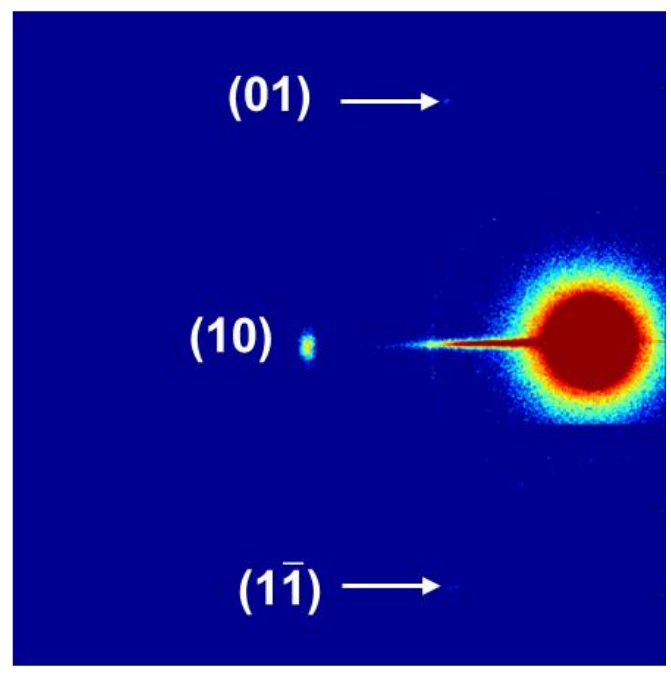

Figure S5. a) AFM height image and b) GI-XRD pattern of a P1 thin film spin cast on the silicon substrate after annealing at $110^{\circ} \mathrm{C}$ for $12 \mathrm{~h}$. 
a)

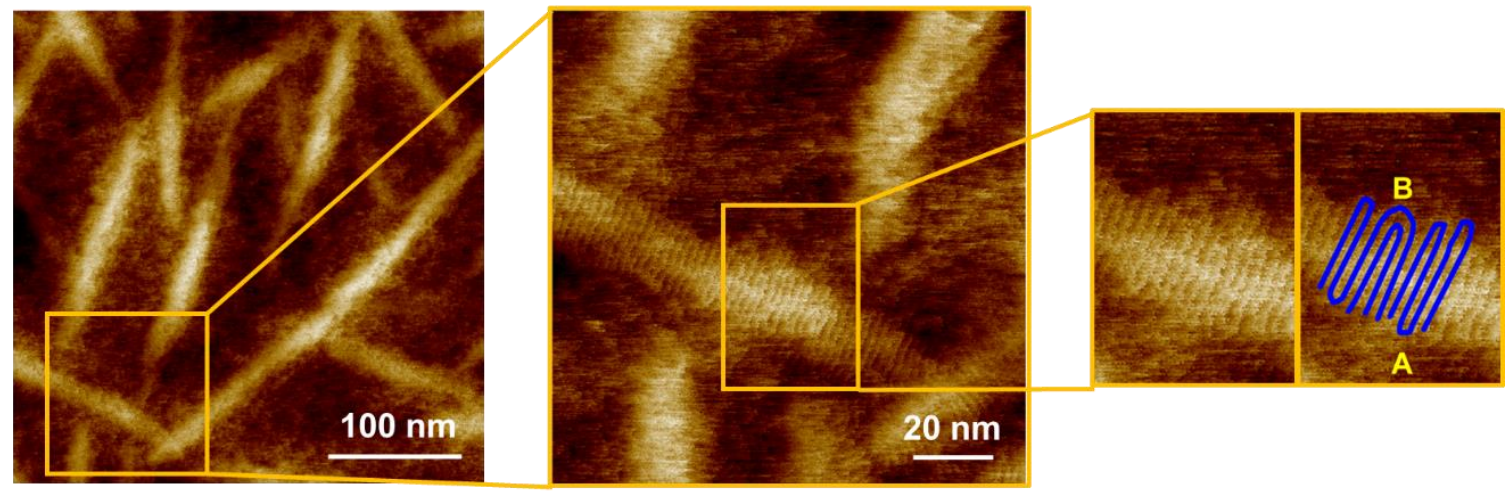

b)

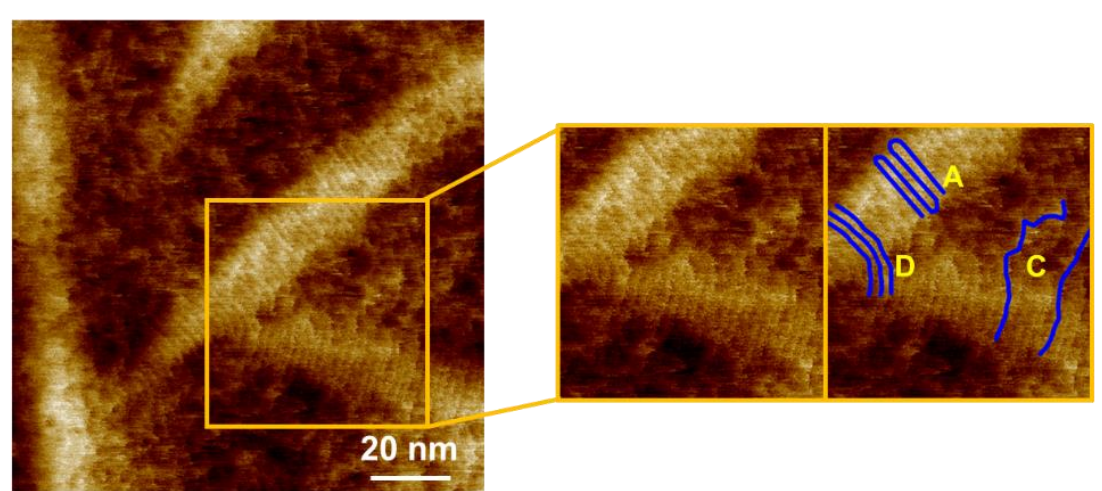

Figure S6. AFM height images of a $\mathbf{P 1}$ thin film spin cast on the silicon substrate after annealing at $120{ }^{\circ} \mathrm{C}$ for $2 \mathrm{~h}$. In a) and b), the areas of the zoomed images shown in Figure $2 \mathrm{c}$ and $2 \mathrm{e}$ of the main text are indicated, respectively. 
a)

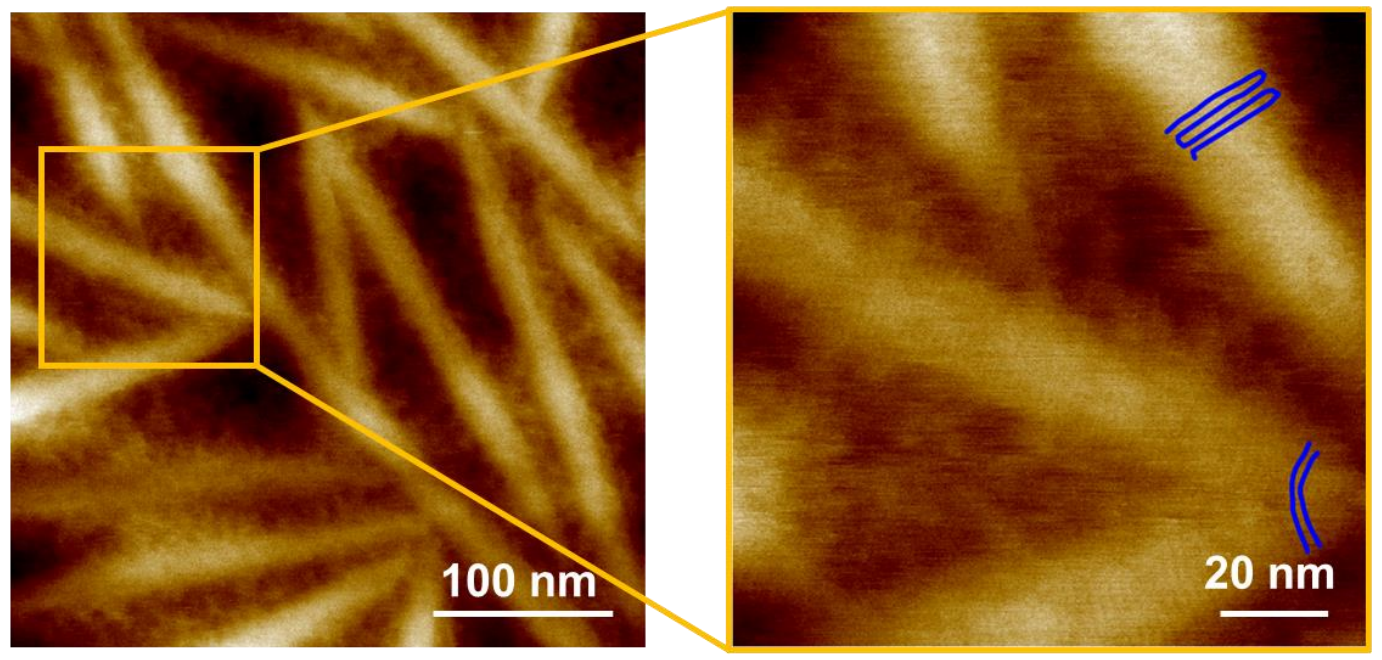

b)

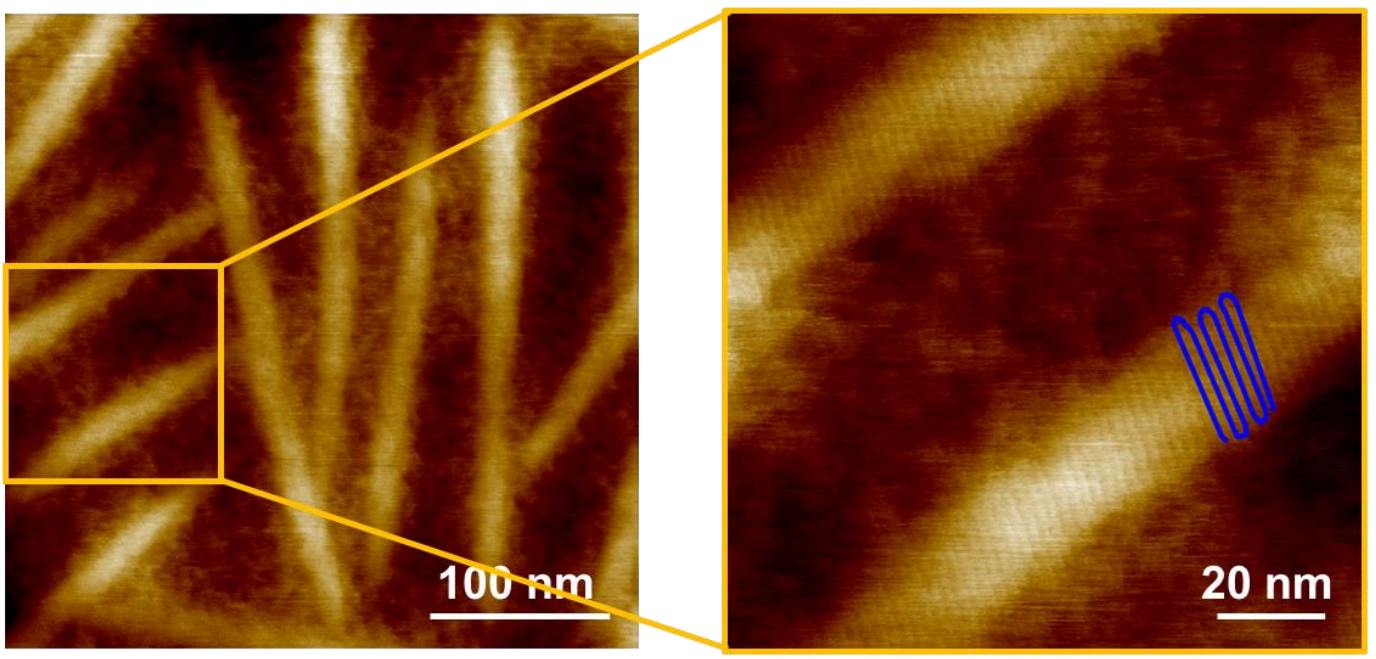

c)

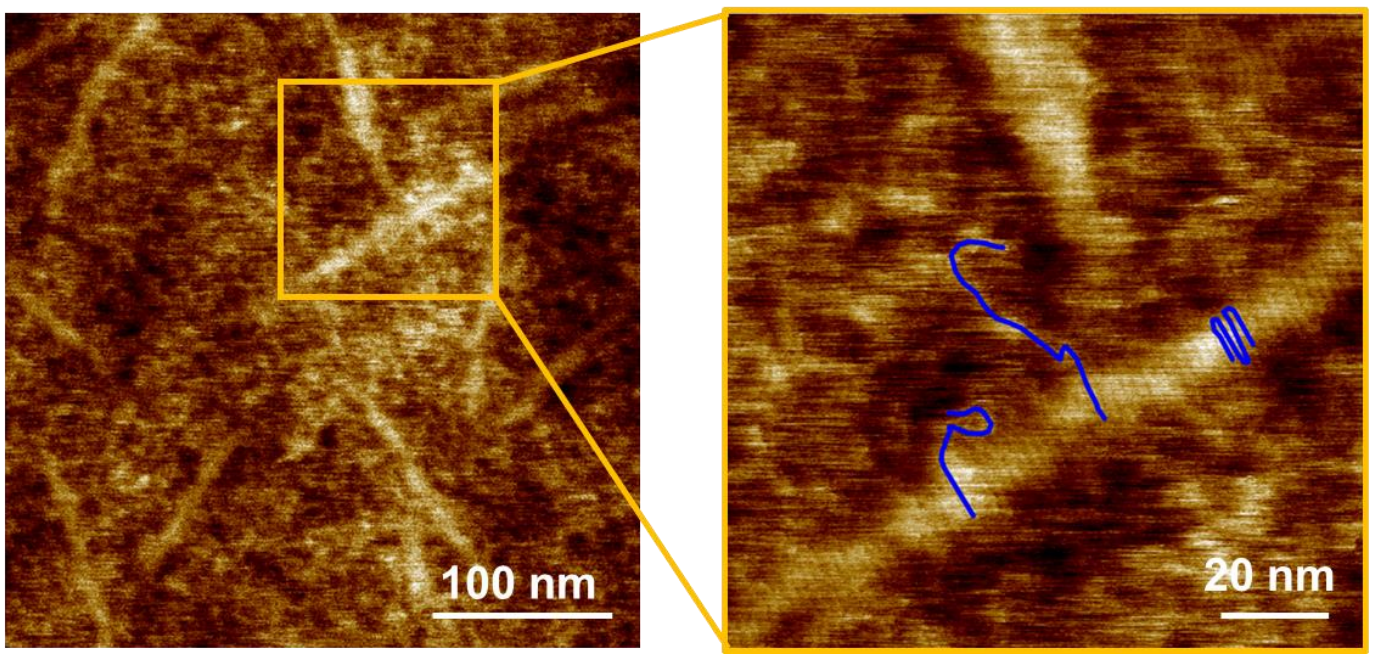

Figure S7. AFM height images and zoomed images of a P1 thin film spin cast on the silicon substrate after annealing at $110{ }^{\circ} \mathrm{C}$ for a) 1 , b) 12 and c) $24 \mathrm{~h}$, respectively. 

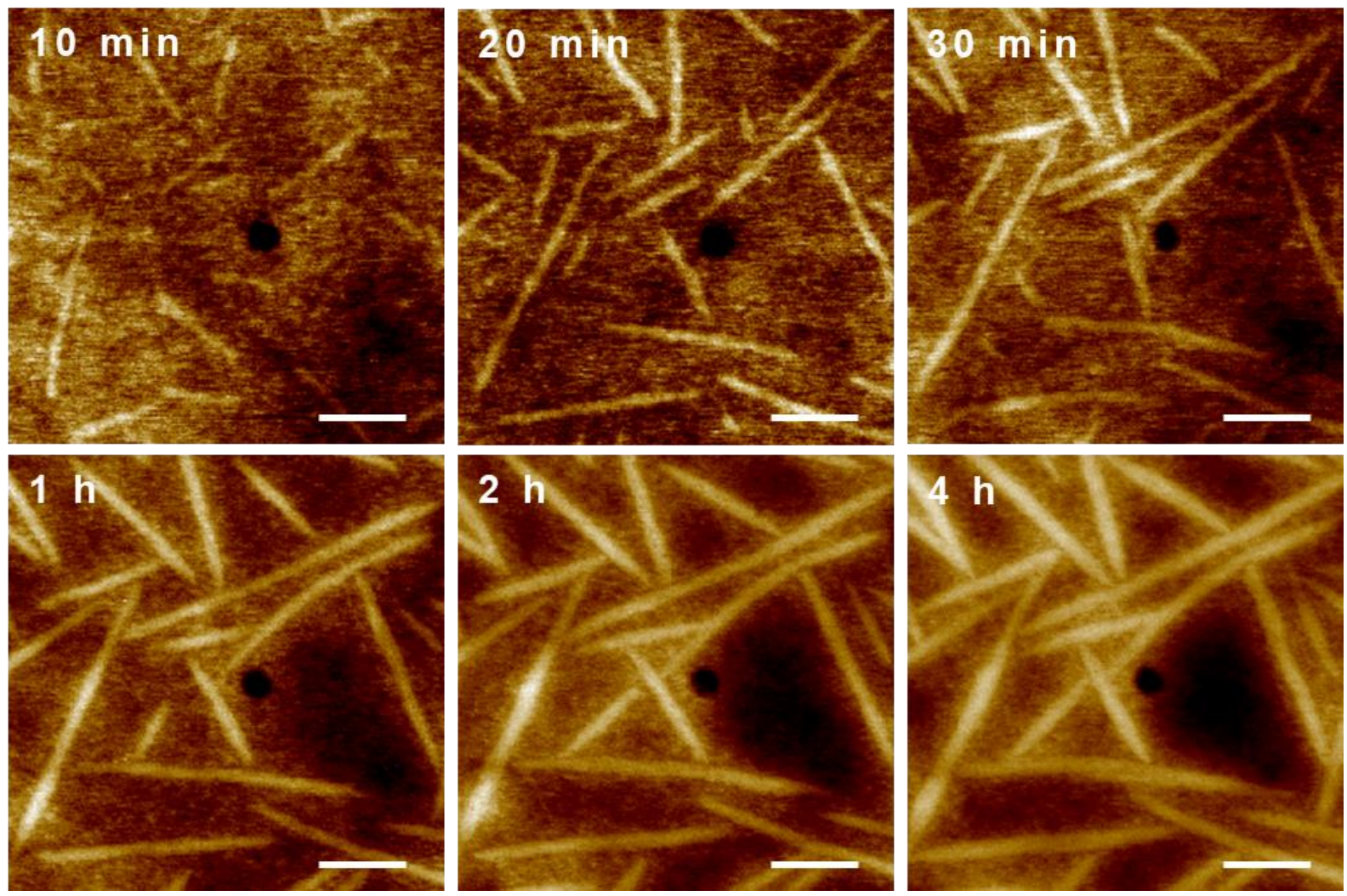

Figure S8. Quasi-in-situ AFM height images of a P1 thin film spin cast on the silicon substrate annealed at $120^{\circ} \mathrm{C}$ for different times. Scale bar: $100 \mathrm{~nm}$. 
a)
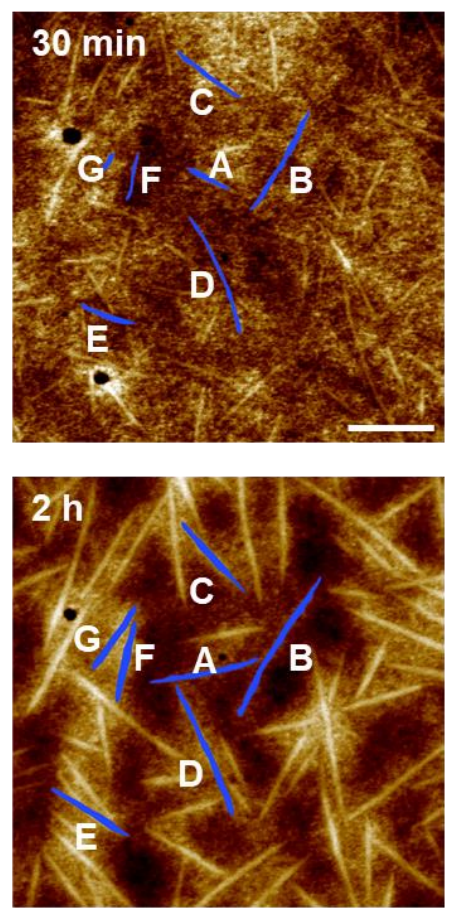
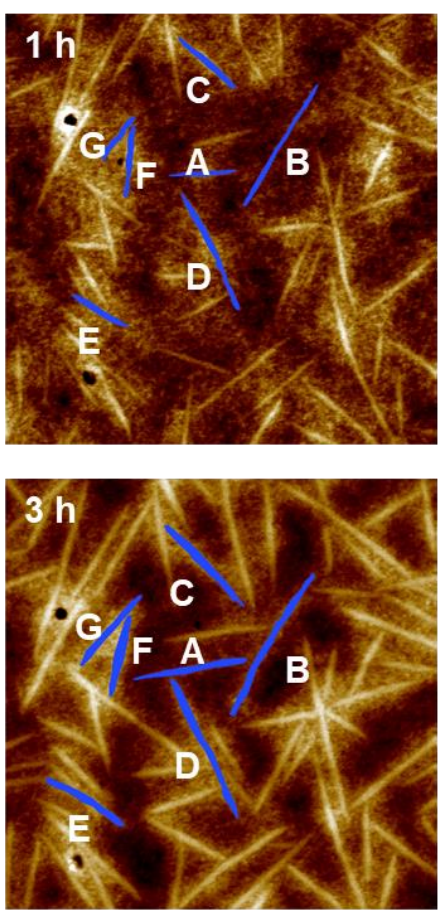
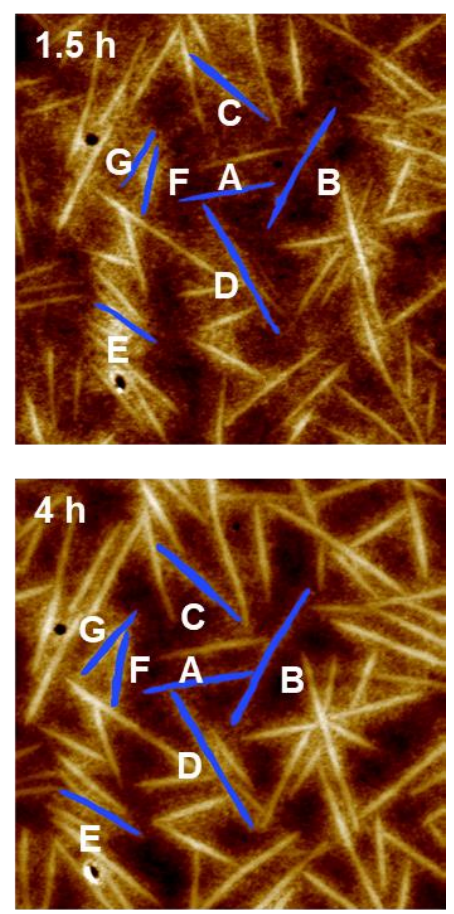

b)

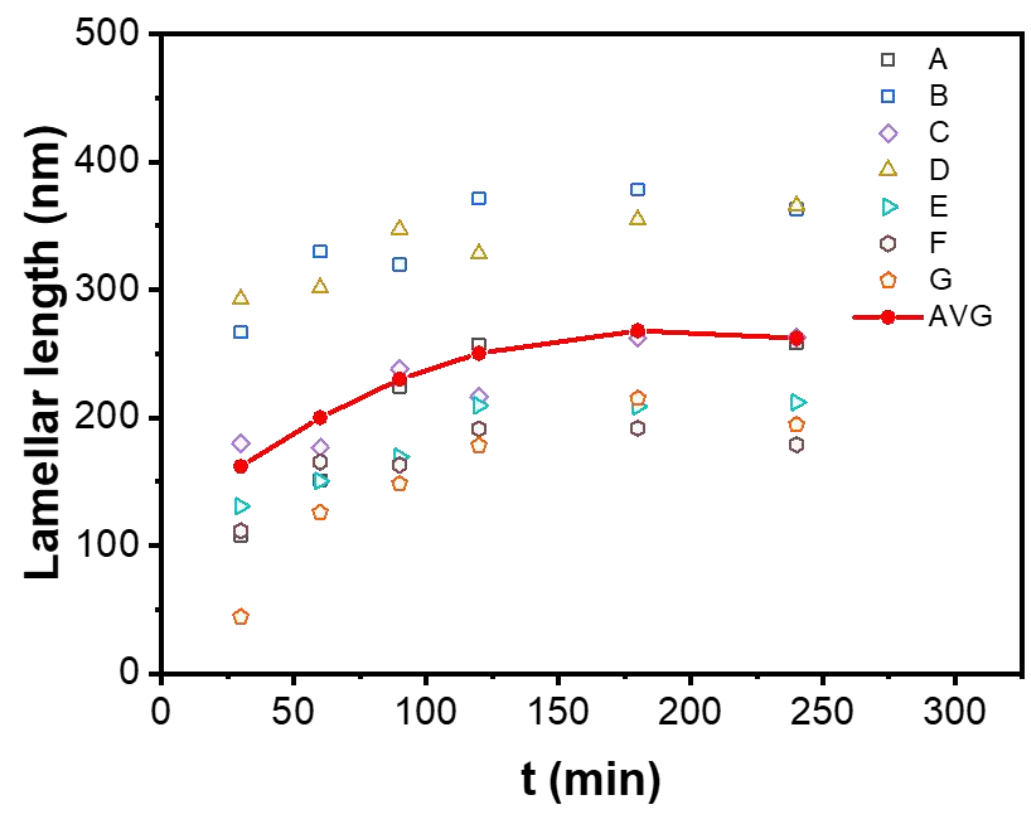

Figure S9. a) Quasi-in-situ AFM height images of a $\mathbf{P 1}$ thin film spin cast on the silicon substrate annealing at $110{ }^{\circ} \mathrm{C}$ for different times. The lamellae selected for the length measurement, which can grow freely towards both sides in the early stage, are denoted as A G. Scale bar: $200 \mathrm{~nm}$. b) Plot of lamellar lengths vs. annealing time. As can be seen, the lengths of lamellae A-G are scattered, which should be due to that the lamellae started to grow at different times. Nonetheless, the growth tendencies of $A-G$ are the same. The solid red dots represent the mean lamellar length (i.e., $W$ in the main text), which was used for the analysis of the Ostwald ripening kinetics. 
a)
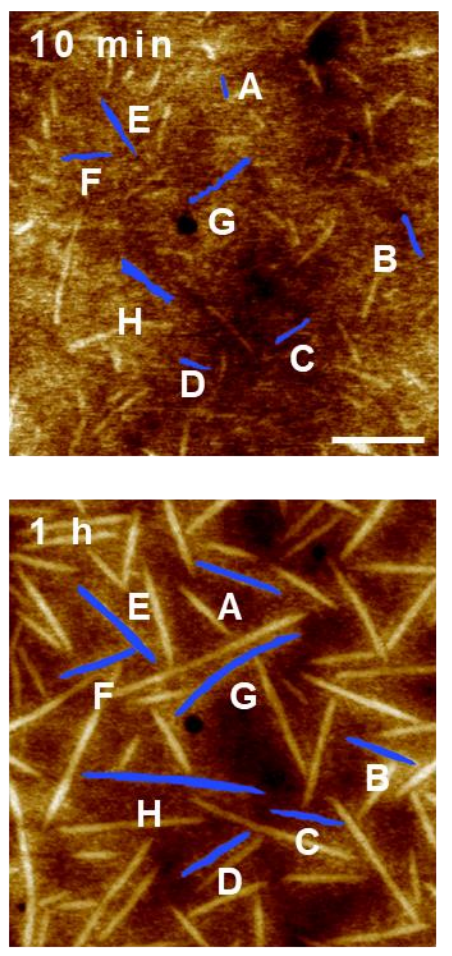
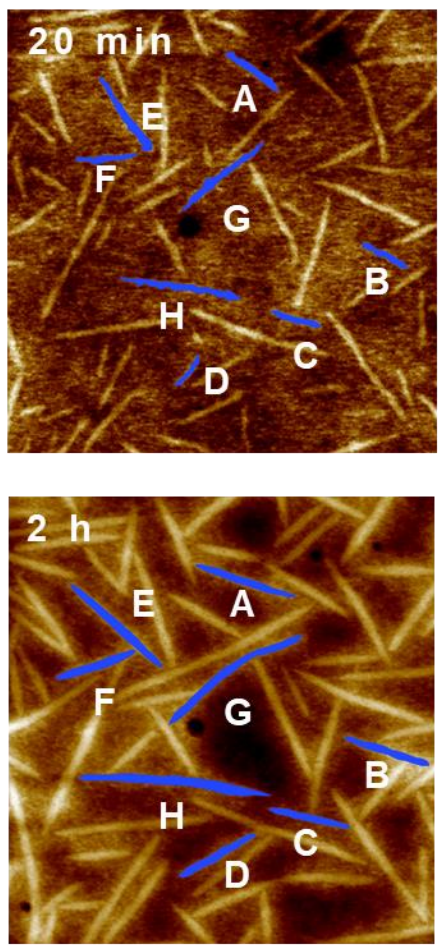
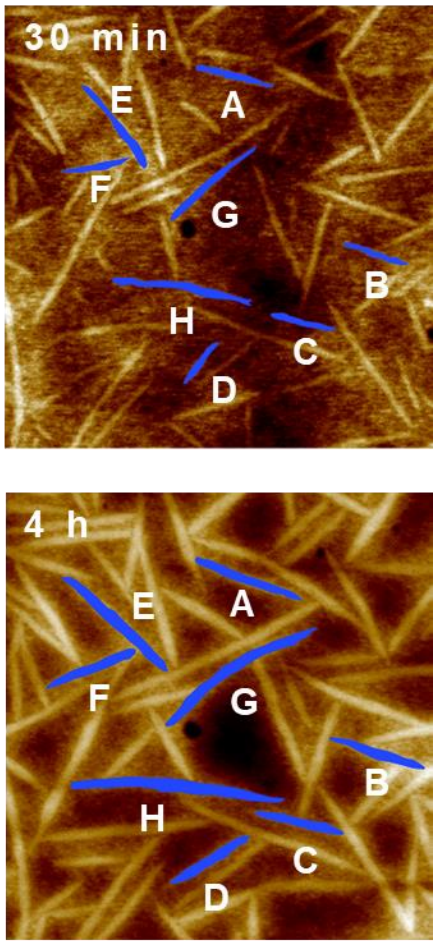

b)

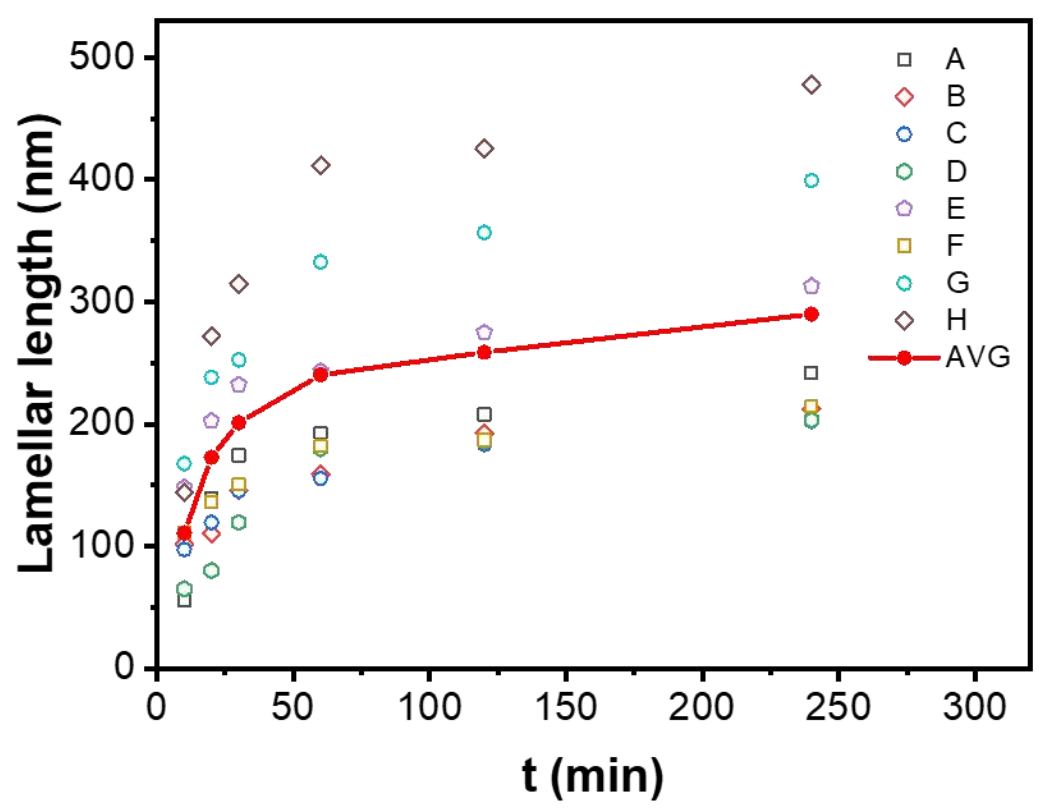

Figure S10. a) Quasi-in-situ AFM height images of a P1 thin film spin cast on the silicon substrate annealed at $120{ }^{\circ} \mathrm{C}$ for different times. The lamellae selected for the length measurement, which can grow freely towards both sides in the early stage, are denoted as A H. Scale bar: $200 \mathrm{~nm}$. b) Plot of lamellar length vs. annealing time. The lengths of A - H in a) are presented. The solid red dots represent the mean lamellar length (i.e., $W$ in the main text), which was used for the analysis of the Ostwald ripening kinetics. As at $120^{\circ} \mathrm{C}$ the lamellae grew faster than those at $110{ }^{\circ} \mathrm{C}$, the lamellae could interfer with each other earlier during growth. 

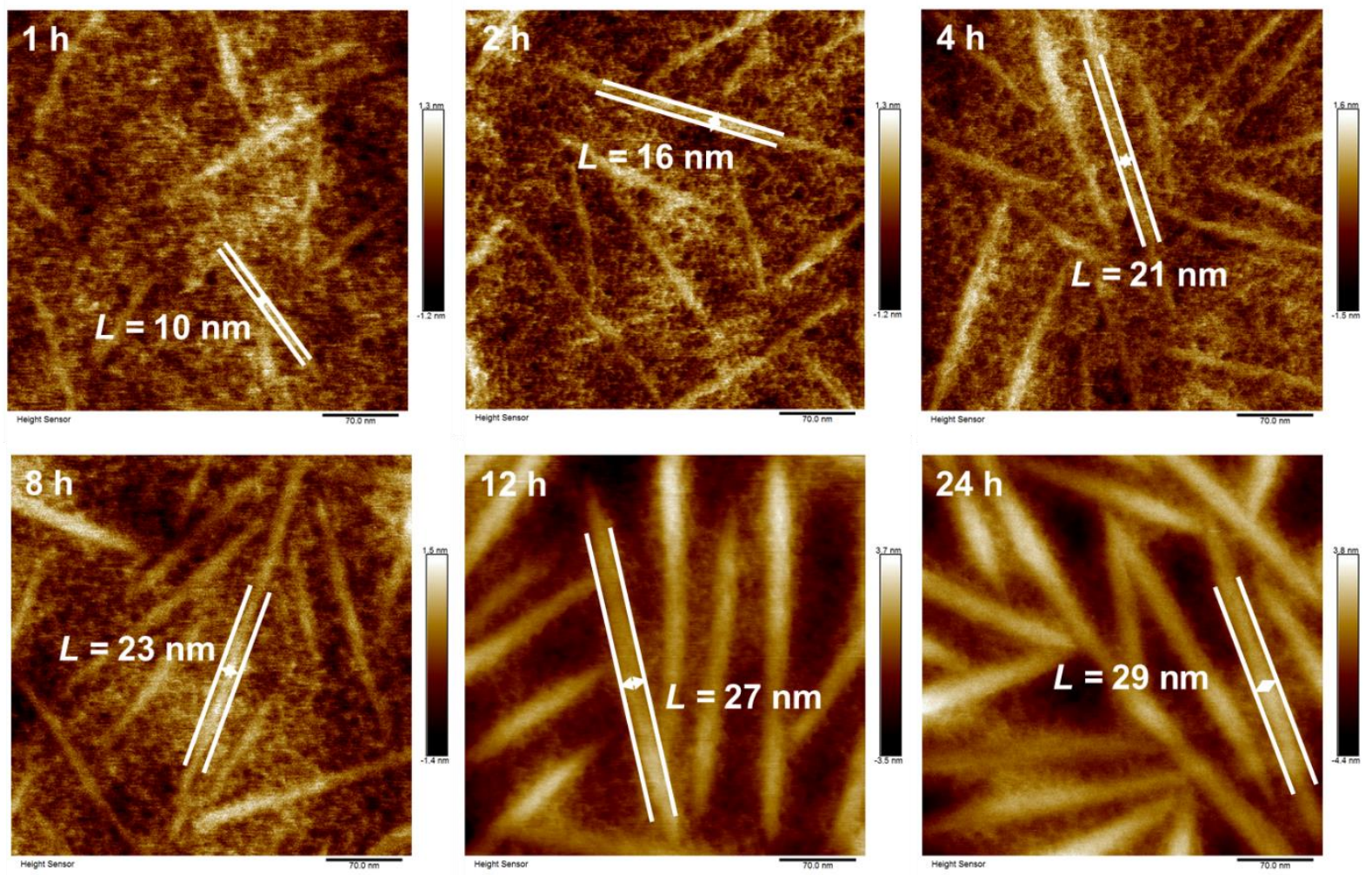

Figure S11. Ex-situ AFM height images of a P1 thin film spin cast on the silicon substrate annealed at $110{ }^{\circ} \mathrm{C}$ for different times. Scale bar: $70 \mathrm{~nm}$. The parallel white lines indicate the edges at the center portion of the lamella based on personal judgment, which we used to measure the lamellar thickness. The mean lamellar thickness (i.e., $L$ in the main text) at a given annealing time was obtained from 15 - 20 lamellae. 

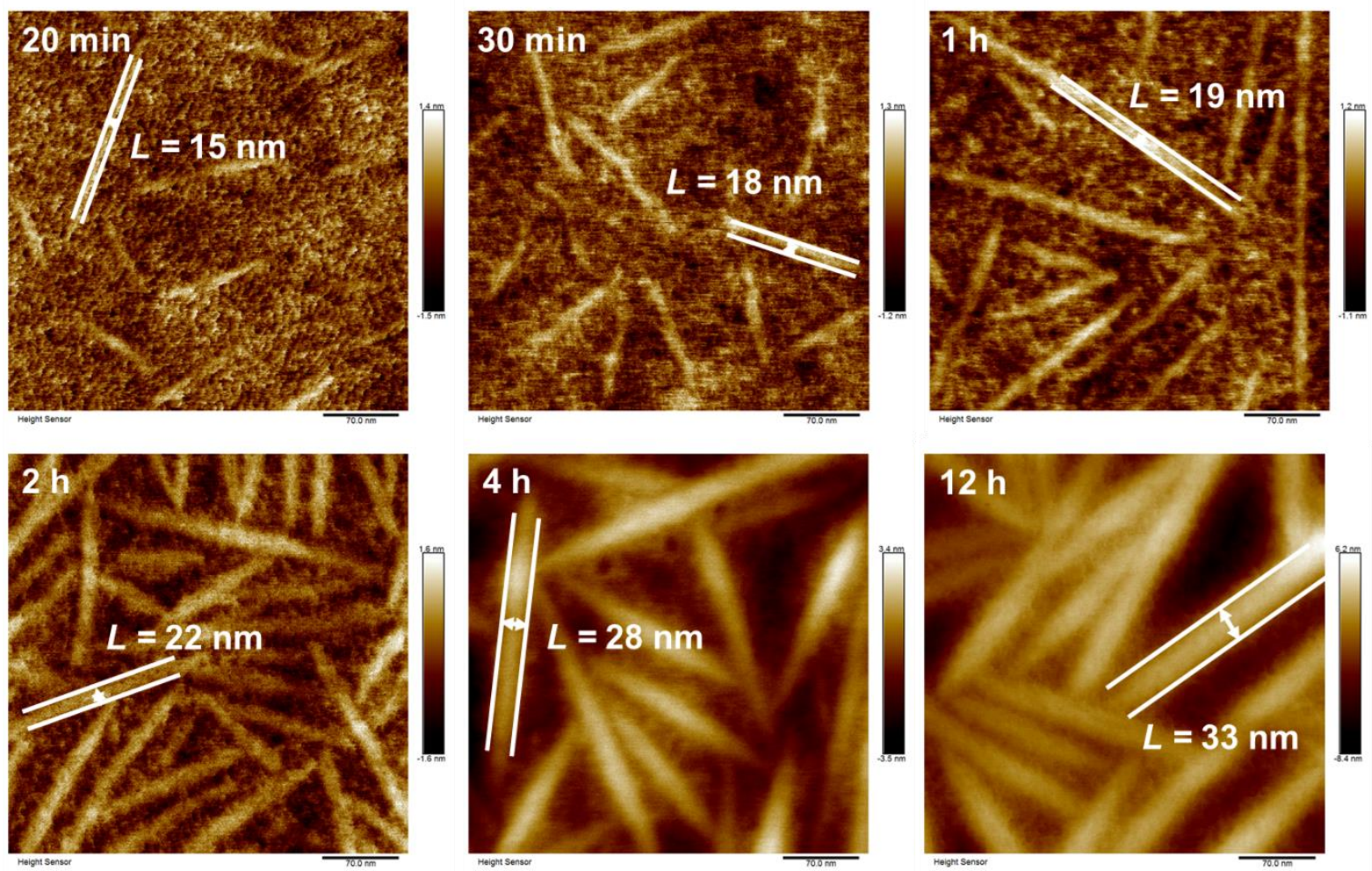

Figure S12. Ex-situ AFM height images of a P1 thin film spin cast on the silicon substrate annealed at $120{ }^{\circ} \mathrm{C}$ for different times. Scale bar: $70 \mathrm{~nm}$. The parallel white lines indicate the edges at the center portion of the lamella based on personal judgment, which we used to measure the lamellar thickness. The mean lamellar thickness (i.e., $L$ in the main text) at a given annealing time was obtained from $15-20$ lamellae. 
a)
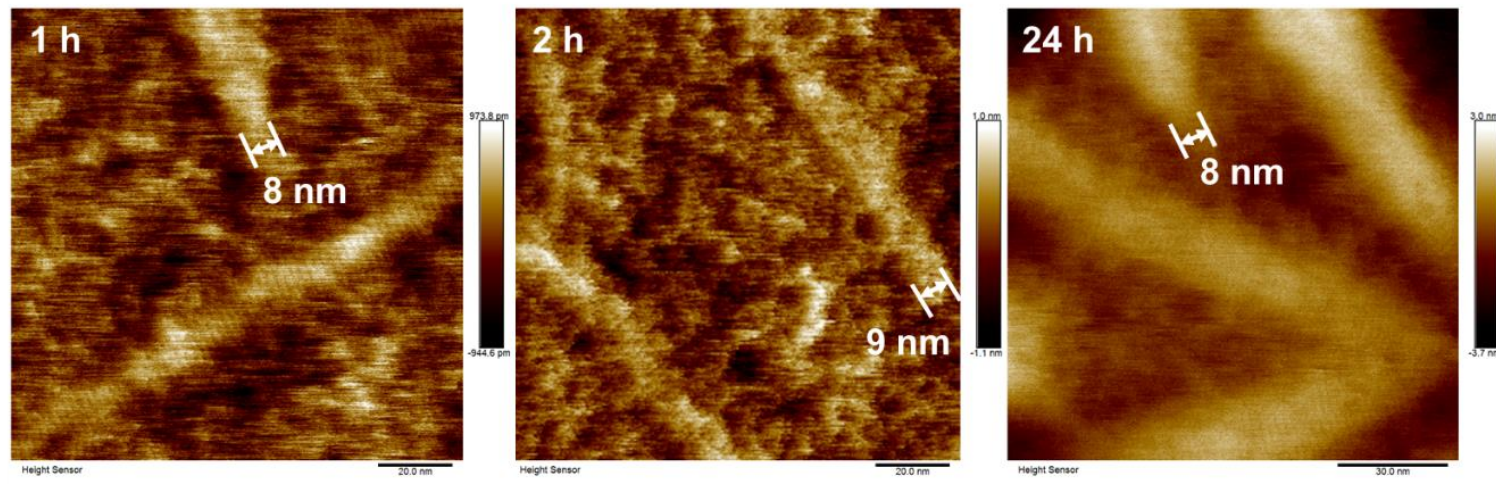

b)
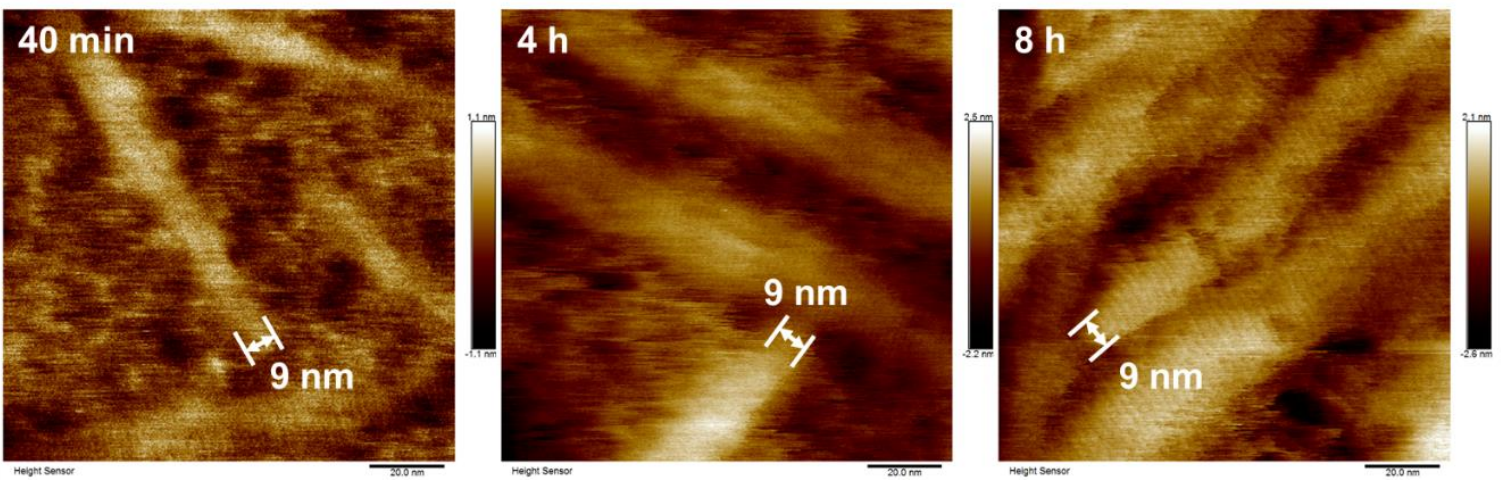

Figure S13. Ex-situ AFM height images of P1 thin films spin cast on the silicon substrate annealed at a) 110 and b) $120^{\circ} \mathrm{C}$ for different times. Scale bar: $20 \mathrm{~nm}$. The double-sided arrows indicate the width of lamellar growth tips. To measure the tip width, high resolution AFM images were used. The mean tip width reported in the main text was based on the measurement of 15 - 20 lamellae grown at the given annealing temperature with different annealing times. 


\section{Synthesis and Molecular Characterization}

The chemical structures and synthetic routes of monomer M1 and polymer P1 are shown in Scheme S1. The experimental details are described as follows.

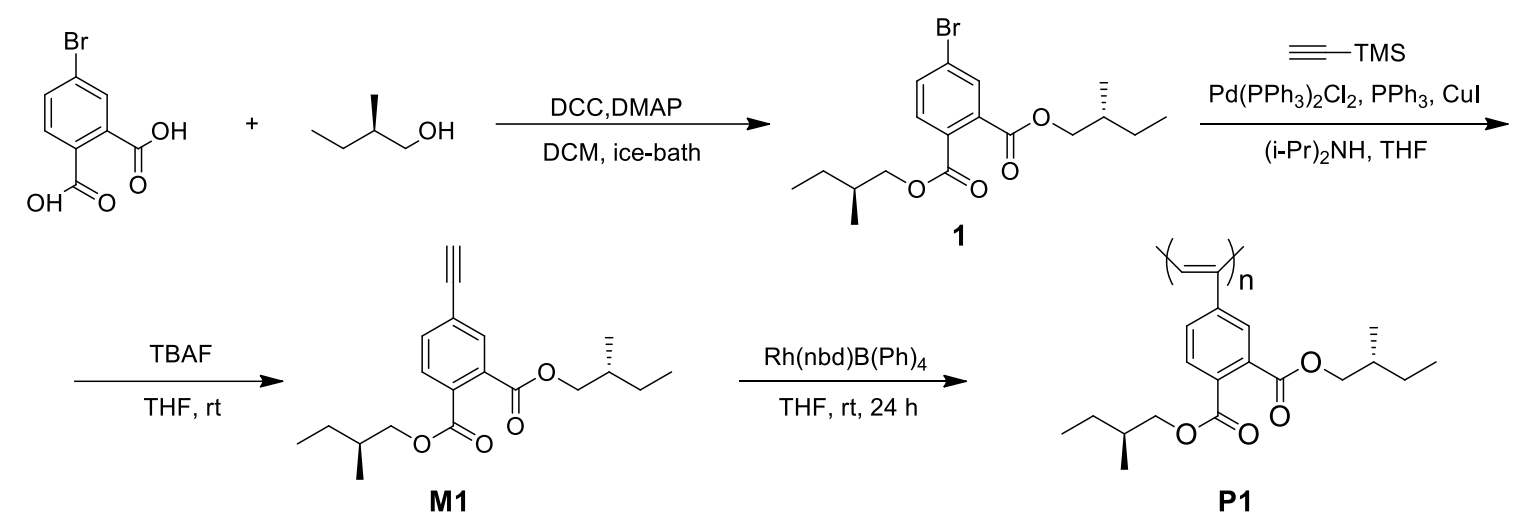

Scheme S1. Synthetic routes to $\mathbf{P 1}$.

\section{Synthesis of bis((S)-2-methylbutyl) 4-bromophthalate (1)}

To a solution of 4-bromophthalic acid (2.45 g, $0.01 \mathrm{~mol})$, 4-dimethylaminopyridine (DMAP, $0.37 \mathrm{~g}, 0.003 \mathrm{~mol}$ ) and (S)-2-methylbutan-1-ol (1.94 g, $0.022 \mathrm{~mol}$ ) in dichloromethane (DCM, $50 \mathrm{~mL}$ ), under the ice bath, was added N,N'-Dicyclohexylcarbodiimide (DCC) (6.19 g, $0.03 \mathrm{~mol}$ ) and stirred at room temperature for $12 \mathrm{~h}$. The mixture was then filtered to remove the insoluble salt. After removing the solvent, the crude product was purified by column chromatography using petroleum ether/ethyl acetate $(25 / 1, \mathrm{v} / \mathrm{v})$ as the eluent. Bis((S)-2methylbutyl) 4-bromophthalate (1) was obtained as a colorless liquid in yield of 59\%. ${ }^{1} \mathrm{H}$ NMR $\left(400 \mathrm{MHz}, \mathrm{CDCl}_{3}, \mathrm{ppm}\right) \delta 7.81(\mathrm{~d}, \mathrm{~J}=1.9 \mathrm{~Hz}, \mathrm{ArH}, 1 \mathrm{H}), 7.65(\mathrm{dt}, \mathrm{J}=17.3,5.1 \mathrm{~Hz}, \mathrm{ArH}, 2 \mathrm{H})$, $4.13\left(\mathrm{~m}, \mathrm{COOCH}_{2}, 4 \mathrm{H}\right), 1.89-1.74\left(\mathrm{~m}, \mathrm{COOCH}_{2} \mathrm{CH}, 2 \mathrm{H}\right), 1.55-1.17\left(\mathrm{~m}, \mathrm{CH}_{2} \mathrm{CH}_{3}, 4 \mathrm{H}\right), 1.03$ - $0.88\left(\mathrm{~m}, \mathrm{CH}_{3}, 12 \mathrm{H}\right)$.

\section{Synthesis of bis((S)-2-methylbutyl) 4-ethynylphthalate (M1)}

1 (1.5 g, $4 \mathrm{mmol}),\left[\mathrm{Pd}\left(\mathrm{PPh}_{3}\right)_{2} \mathrm{Cl}_{2}\right]$ (54.6 mg, $\left.0.078 \mathrm{mmol}\right), \mathrm{CuI}(7.4 \mathrm{mg}, 0.039 \mathrm{mmol}$ ), and $\mathrm{Ph}_{3} \mathrm{P}(20.4 \mathrm{mg}, 0.078 \mathrm{mmol})$ were added to a $50 \mathrm{~mL}$ Schlenk tube with a sidearm. The tube was evacuated under vacuum and then flushed with dry nitrogen three times through the sidearm. DIPA and THF $(15 \mathrm{~mL} / 5 \mathrm{ml})$ were injected into the tube. Ethynyltrimethylsilane $(0.48 \mathrm{~g}, 4.87$ mmol) was then added to the mixture under nitrogen. After stirring for $12 \mathrm{~h}$ at $85^{\circ} \mathrm{C}$, the mixture was cooled to room temperature and filtered to remove the insoluble salt. The salt was washed with triethylamine and ethyl ether until the ether washings were clear. The combined filtrates 
were reduced to dryness under reduced pressure. After that, to the round-bottom flask was directly added THF (50 mL) and tetra-n-butylammonium fluoride (TBAF, $1.4 \mathrm{~g}, 5.4 \mathrm{mmol}$ ). The mixture was stirred at room temperature for 30 minutes. Water was added to quench the reaction. After removing the solvent, the crude product was purified by column chromatography using petroleum ether/ethyl acetate (25/1, v/v) as the eluent. M1 was obtained as a colorless liquid in yield of 68\%. ${ }^{1} \mathrm{H} \mathrm{NMR}\left(400 \mathrm{MHz}, \mathrm{CDCl}_{3}, \mathrm{ppm}\right) \delta 7.80(\mathrm{~d}, \mathrm{~J}=1.5 \mathrm{~Hz}, \mathrm{ArH}, 1 \mathrm{H}), 7.72$ - 7.59 (m, $\mathrm{ArH}, 2 \mathrm{H}), 4.24$ - 4.03 (m, $\left.\mathrm{COOCH}_{2}, 4 \mathrm{H}\right), 3.23$ (s, $\left.\equiv \mathrm{CH}, 1 \mathrm{H}\right), 1.88$ - 1.76 (m, $\left.\mathrm{COOCH}_{2} \mathrm{CH}, 2 \mathrm{H}\right), 1.55-1.17\left(\mathrm{~m}, \mathrm{CH}_{2} \mathrm{CH}_{3}, 4 \mathrm{H}\right), 1.00-0.91\left(\mathrm{~m}, \mathrm{CH}_{3}, 12 \mathrm{H}\right)$. HRMS (ESI positive): $[\mathrm{M}+\mathrm{Na}]^{+}$calcd for $\mathrm{C}_{20} \mathrm{H}_{26} \mathrm{NaO}_{4} \mathrm{~m} / \mathrm{z}$ 353.17233, found 353.17272. Anal. calcd for $\mathrm{C}_{20} \mathrm{H}_{26} \mathrm{O}_{4}$ : C, 72.70; H, 7.93; found: C, 72.68; H, 7.97.

\section{Synthesis of P1}

Into a $10 \mathrm{~mL}$ Schlenk tube with a sidearm was added M1 (330 mg, $1 \mathrm{mmol})$. The tube was evacuated under vacuum and then flushed with dry nitrogen three times through the sidearm. THF (3 mL) was injected into the tube to dissolve the monomer. A solution of the rhodium catalyst was prepared in another tube by dissolving $5.2 \mathrm{mg}, 0.01 \mathrm{mmol}$ of $\mathrm{Rh}(\mathrm{nbd})\left[\mathrm{B}\left(\mathrm{C}_{6} \mathrm{H}_{5}\right)_{4}\right]$ in $2 \mathrm{ml}$ THF which was transferred to the monomer solution using a hypodermic syringe at room temperature. The concentrations of the monomer and the rhodium catalyst were $0.2 \mathrm{M}$ and $0.002 \mathrm{M}$, respectively. The polymerization rapidly proceeded and the color turned to darkorange within a few seconds. After $24 \mathrm{~h}$ at $30^{\circ} \mathrm{C}$, the mixture was then diluted with $5 \mathrm{~mL}$ of THF and added dropwise to a mixture of methanol $(150 \mathrm{~mL})$ under stirring. The resulting polymer P1 was precipitated, collected by centrifugation, and dried in a vacuum oven at room temperature to a constant weight. The orange solid was obtained in $98 \%$ yield. ${ }^{1} \mathrm{H}$ NMR (400 $\left.\mathrm{MHz}, \mathrm{CDCl}_{3}, \mathrm{ppm}\right) \delta 7.37$ (d, J = 6.5 Hz, $\left.\mathrm{ArH}, 1 \mathrm{H}\right), 7.25$ (s, $\left.\mathrm{Ar} H, 1 \mathrm{H}\right), 6.75$ (d, J = 4.8 Hz, ArH, 1H), $5.71(\mathrm{~s},=\mathrm{CH}, 1 \mathrm{H}), 3.94\left(\mathrm{~m}, \mathrm{COOCH}_{2}, 4 \mathrm{H}\right), 1.79-1.49\left(\mathrm{~m}, \mathrm{COOCH}_{2} \mathrm{CH}, 2 \mathrm{H}\right), 1.45$ - 0.98 (m, $\left.\mathrm{CH}_{2} \mathrm{CH}_{3}, 4 \mathrm{H}\right), 0.91-0.69\left(\mathrm{~m}, \mathrm{CH}_{3}, 12 \mathrm{H}\right)$. 


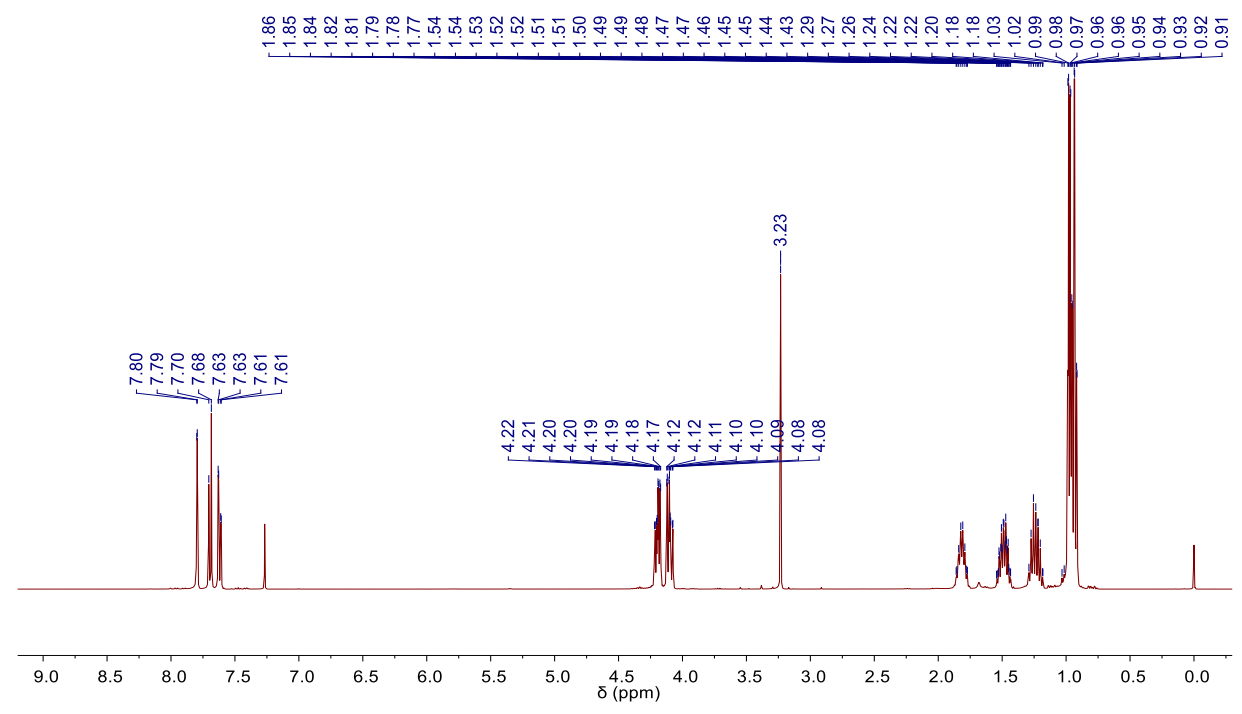

Figure S14. ${ }^{1} \mathrm{H} \mathrm{NMR}$ spectra of $\mathrm{M1}$ in $\mathrm{CDCl}_{3}$.

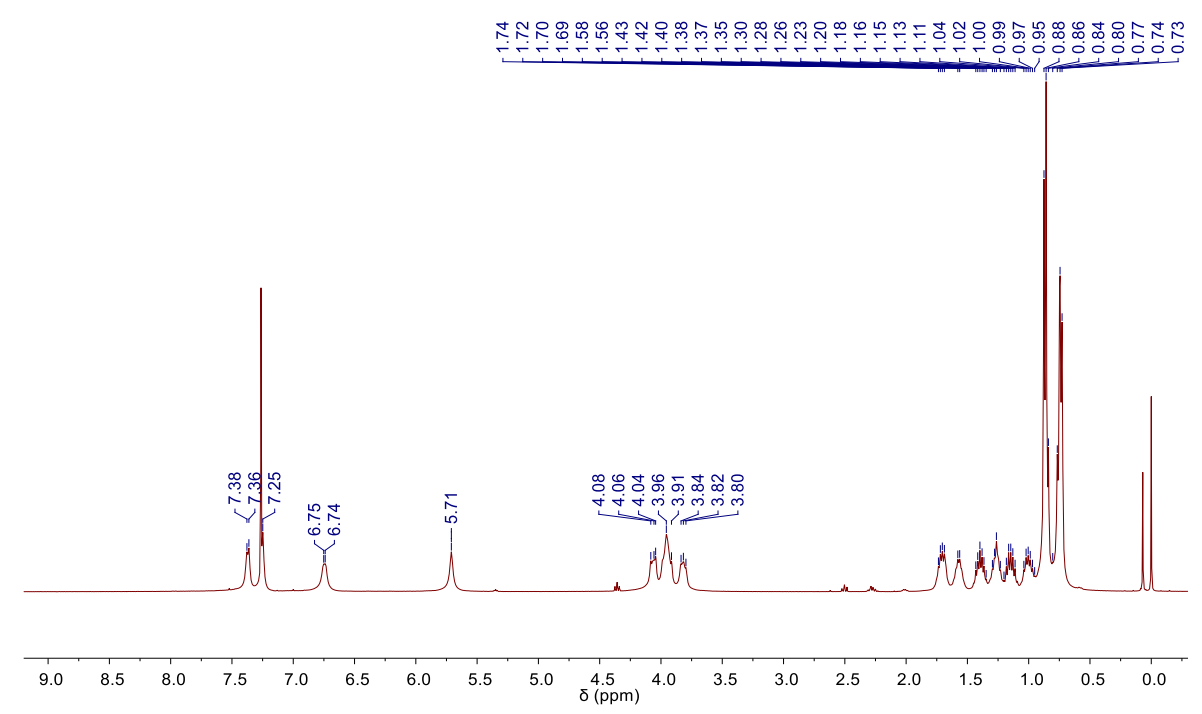

Figure S15. ${ }^{1} \mathrm{H}$ NMR spectra of $\mathbf{P 1}$ in $\mathrm{CDCl}_{3}$.

Table S1. Polymerization results of $\mathbf{P 1}$.

\begin{tabular}{ccccccc}
\hline Sample & $\begin{array}{c}M_{\mathrm{n}, \mathrm{GPC}} \\
\left(\times 10^{4} \mathrm{~g} / \mathrm{mol}\right)\end{array}$ & $\begin{array}{c}M_{\mathrm{w}, \mathrm{GPC}} \\
\left(\times 10^{4} \mathrm{~g} / \mathrm{mol}\right)\end{array}$ & PDI & $\begin{array}{c}\text { Yield } \\
(\%)\end{array}$ & $\begin{array}{c}\text { Cis-content }^{a} \\
(\%)\end{array}$ & $\begin{array}{c}M_{\mathrm{w}, \mathrm{SLS}} \\
\left(\times 10^{4} \mathrm{~g} / \mathrm{mol}\right)\end{array}$ \\
\hline P1 & 16.6 & 29.0 & 1.75 & 98 & 90 & 141.0 \\
\hline
\end{tabular}

${ }^{a}$ The cis-content can be estimated by $c i s \%=\frac{A_{H} \times 4}{A_{H}+A_{P h}} \times 100$, where $\mathrm{A}_{\mathrm{H}}$ and $\mathrm{A}_{\mathrm{Ph}}$ are the area integrals of the olefin proton and aromatic proton, respectively. 


\section{References}

(1) Schrock, R. R.; Osborn, J. A. $\pi$-Bonded Complexes of the Tetraphenylborate Ion with Rhodium(I) and Iridium(I). Inorg. Chem. 1970, 9, 2339-2343.

(2) Yao, J. H.; Elder, K. R.; Guo, H.; Grant, M. Theory and Simulation of Ostwald Ripening. Phys. Rev. B 1993, 47, 14110-14125. 